\title{
Computer simulation of thin film growth: applying the results to optical coatings
}

\section{Sikkens}

University of Groningen

Department of Applied Physics

Nijenborgh 18

9747 AG Groningen, The Netherlands

\section{J. Hodgkinson}

University of Otago

Department of Physics

Dunedin, New Zealand

\section{F. Horowitz}

Universidade do Rio Grande do Sul

Instituto de Fisica

90000 Porto Alegre, RS Brazil

\section{H. A. Macleod}

Optical Sciences Center

University of Arizona

Tucson, Arizona 85721

\section{J. J. Wharton}

Air Force Institute of Technology

Wright-Patterson AFB, Ohio 45433

\begin{abstract}
Computer simulation of thin film growth has been used extensively to gain insight into the origin and nature of the microstructure of vapor-deposited thin films. Usually, however, no attempts are made to predict film properties other than column angle and film density from such simulations. The aim of our work is to derive quantitative data from computer simulations in order to be able to predict relevant properties of optical coatings. The deposition of 2500 to 25,000 particles has been simulated on different computers by random deposition of two-dimensional hard disks, using a simple relaxation scheme. Statistical analysis of the results yields quantitative data for the density, column angle, and column period. On the basis of these results, a simple model has been developed for the microstructure of a three-dimensional film. The birefringence and the shape of water-penetration fronts in evaporated optical coatings, predicted from this model, are confirmed by experiment.
\end{abstract}

Subject terms: thin films; crystal growth; optical coatings.

Optical Engineering 25(1), 142-147 (January 1986).

\section{CONTENTS}

1. Introduction

2. Computer simulation

3. Statistical analysis of simulated films 3.1. Film density

3.2. Column orientation

3.3. Periodicity

4. Simplified model for the microstructure of a threedimensional film

5. Water penetration in obliquely deposited films

6. Form birefringence in thin films

7. Conclusions

8. Acknowledgments

9. References

\section{INTRODUCTION}

In vapor deposition processes used to fabricate thin films, the low condensation temperature leads to a film structure consisting of loosely packed columns separated by voids. ${ }^{1}$ This

Paper 2051 received July 30, 1984; revised manuscript received April 29, 1985; accepted for publication May 3, 1985; received by Managing Editor June 17, 1985. This paper is a revision of Paper 505-48 which was presented at the SPIE conference on Advances in Optical Materials, Aug. 21-24, 1984, San Diego, Calif. The paper presented there appears (unrefereed) in SPIE Proceedings Vol. 505 .

$\odot 1986$ Society of Photo-Optical Instrumentation Engineers. microstructure has a dramatic effect on the magnetic, ${ }^{2-4}$ optical, ${ }^{5-7}$ electrical, $5,6,8-10$ and mechanical ${ }^{11-13}$ properties of thin films.

In optical thin films the porous structure gives rise to internal adsorption of water, which alters the effective refractive index of the material. This leads to drifting of the optical properties of coatings. Furthermore, the essentially anisotropic nature of the columnar microstructure causes form birefringence in optical coatings.

It is evident that a good understanding of the origin of the columnar structure is important when attempts are made to improve the coating quality. The fact that the principal features of the microstructure are common to a wide variety of materials, regardless of chemical composition, suggests an almost purely physical origin.

Computer simulations of the deposition of thin films have contributed significantly to a better understanding of the relevant factors in the deposition process. Essentially, these simulations are based on a model in which atoms or molecules arrive randomly on a surface and interact with the particles that are already deposited. These simulations have been carried out in two dimensions ${ }^{14}$ and in three dimensions. ${ }^{15}$ In zero-mobility models the particles stick exactly where they land, but usually some kind of limited mobility is introduced by allowing the landed particles to move to a minimum energy 
position. ${ }^{14-16}$ The interaction of the incoming particle with the surface is sometimes described in terms of a hard disk or sphere model, ${ }^{14-16}$ but fully dynamic models also have been used. ${ }^{17}$

Up to now, the results of computer simulations have been used mainly for a qualitative comparison with real films. Apart from some isolated calculations of film densities and semiquantitative observations of the column angle, no serious attempts have been made to perform a detailed, quantitative analysis of the properties of such simulated films. In our work, a first attempt is made in this direction. On the basis of the results of a statistical analysis, a simplified model has been developed for the microstructure of a thin film. The optical birefringence and the shape of water penetration fronts in optical coatings, predicted from this model, are confirmed by experiment.

\section{COMPUTER SIMULATION}

The computer program is based on the random deposition of two-dimensional hard disks onto a "substrate" that is a linear array of disks with the same diameter as the incoming disks. Figure 1 illustrates the different steps in the deposition procedure for a particle (disk) incident at an angle $\alpha$ with the substrate normal. The computer program generates a random $\mathrm{x}$-coordinate and subsequently determines the impact position of the particle. In the final step, the particle relaxes to the

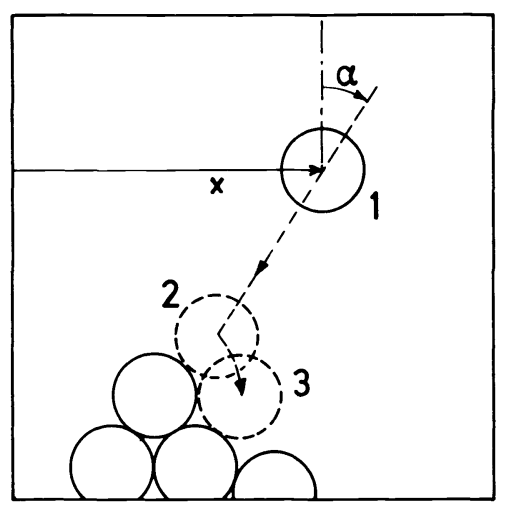

Fig. 1. Illustration of the different steps involved in the computer simulation. (1) The particle starts at a fixed height from the substrate, at a random $x$-coordinate, and follows a trajectory that makes an angle $\alpha$ with the substrate normal. (2) The particle collides with one of the particles on the substrate. (3) The particle "relaxes" to the nearest position at which it touches two others. nearest position at which it touches two other disks. To avoid unrealistic eptiaxial growth, no relaxation occurs if the incident particle hits one of the particles in the "substrate" array.

For computation efficiency, the simulated film segment is organized as a 120 by 64 matrix, each element having a dimension $1 / 2 \sqrt{2}$ by $1 / 2 \sqrt{2}$ in units of the particle diameter. In this way, a matrix element cannot be occupied by more than one particle. Periodic boundary conditions are used for the $\mathrm{x}$-coordinate. The deposition process is terminated if a particle relaxes to a position outside the y-boundary. Typically, about 2500 particles are deposited at normal incidence.

The program is written in PASCAL and has been adapted to run on a M6809 microcomputer and a CYBER 170/760 mainframe. A larger version of the program, with a 640 by 128 matrix, runs on the the CYBER only. This version typically deposits about 25,000 particles at normal incidence. Unless otherwise noted, the results reported here are those obtained with the smaller version of the program.

Figure 2 shows simulated film segments deposited at different angles of incidence that clearly reveal the columnar nature of the microstructure, in particular at large off-normal deposition angles.

\section{STATISTICAL ANALYSIS OF SIMULATED FILMS}

For simplicity, statistical analysis of the film density, the column orientation, and the periodicity is not performed on the exact particle coordinates but instead on a 120 by 64 Boolean matrix that indicates whether or not the corresponding matrix cell is occupied by a particle.

\subsection{Film density}

The film density has been calculated as the average density in rows 8 through 39 of the matrix. These limits are chosen because it turns out that the density, moving away from the substrate, usually drops in the first few rows, then reaches a more or less constant value and drops again as the film/air interface is approached. The density is expressed as a relative quantity with respect to the hexagonal closest packing in two dimensions.

Figure 3 depicts the calculated density as a function of the deposition angle. It is obvious that the density values are considerably lower than those found in real films $(0.80$ to 0.95), which apparently is a consequence of the simulation being two-dimensional and of the relaxation scheme used. Earlier, it was found that the density is even lower for a three-dimensional hard-disk simulation employing the same relaxation scheme. ${ }^{15}$ In the latter case densities of 0.4 to 0.5 are
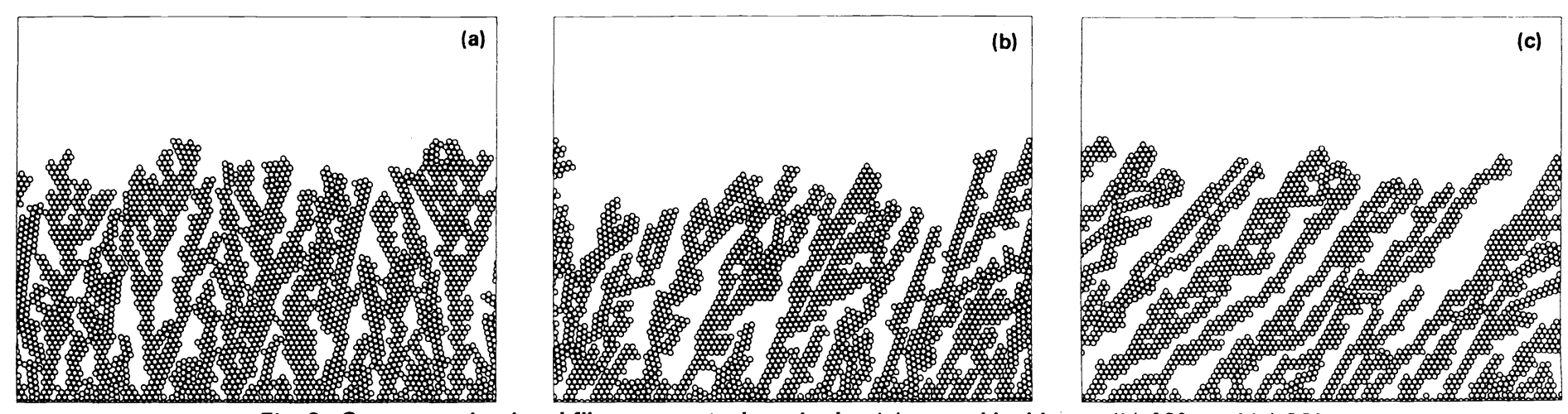

Fig. 2. Computer simulated film segments deposited at (a) normal incidence, (b) $40^{\circ}$, and (c) $60^{\circ}$. 


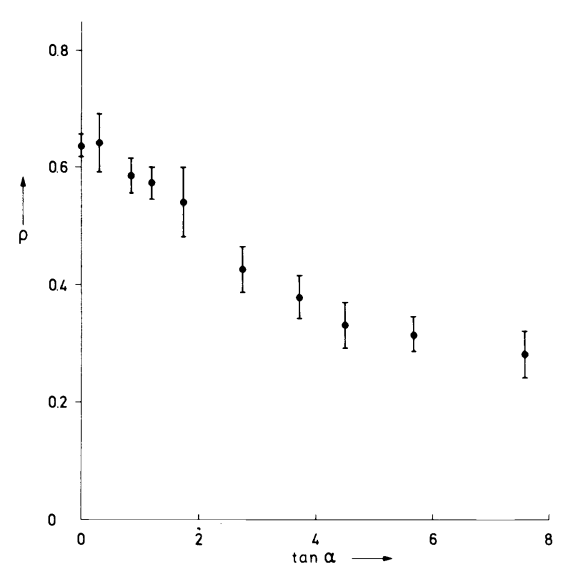

Fig. 3. Density $\rho$ of the computer simulated films versus $\tan \alpha$.

obtained. Therefore, it appears that the main reason for the low density values is the relaxation scheme. Nevertheless, the dependence of the density on the deposition angle still may be realistic. In practical films, it has been observed that the density decreases almost linearly with $\tan \alpha .^{18-19}$ However, an exactly linear relationship, as has been suggested by Leamy, Gilmer, and Dirks, ${ }^{17}$ is unrealistic since the density will eventually become negative at large deposition angles.

It seems more realistic to assume that the density behaves as

$\rho=\frac{1}{p+q \tan \alpha}$.

In Fig. 4, $1 / \rho$ is plotted versus $\tan \alpha$. The figure shows that Eq. (1) appears to be satisfied. In this particular case, the numerical constants are $p=1.50$ and $q=0.291$. It should be realized that the values of the constants will depend strongly on the relaxation scheme, which is not very realistic in this case. However, the general form of Eq. (1) is apparently valid for a wide variety of practical films. ${ }^{17}$

The film density also has been calculated from simulations at normal incidence using the larger CYBER program version. The average density found in that case is $0.615 \pm 0.014$, illustrating the improved statistics of the result.

\subsection{Column orientation}

The average angle $\beta$ of the columns with the substrate normal also can be determined by a statistical analysis of the simulated films. Somewhat arbitrarily, we have used the following quantity to calculate the average angle (see Fig. 5):

$\mathrm{F}(\theta)=\frac{1}{\mathrm{~L} \rho(1-\rho)} \int_{0}^{\mathrm{L}}\left\{\frac{\cos \theta}{\mathrm{T}} \int_{0}^{\mathrm{T} / \cos \theta}[\rho(\mathrm{x}, \mathrm{y})-\rho] \mathrm{dy}\right\}^{2} \mathrm{dx}$,

where $\rho$ is the average density.

For a perfectly periodic columnar film with infinite thickness, dense columns $(\rho=1)$, and empty voids $(\rho=0)$, this function equals 1 if $\theta$ equals the column angle and is zero for all other $\theta$. For this reason we will refer to this function as the "column orientation function." For the films under consideration here we might expect a somewhat broadened peak at the average column angle.

Figure 6 depicts $F(\theta)$ for three different films. It can be

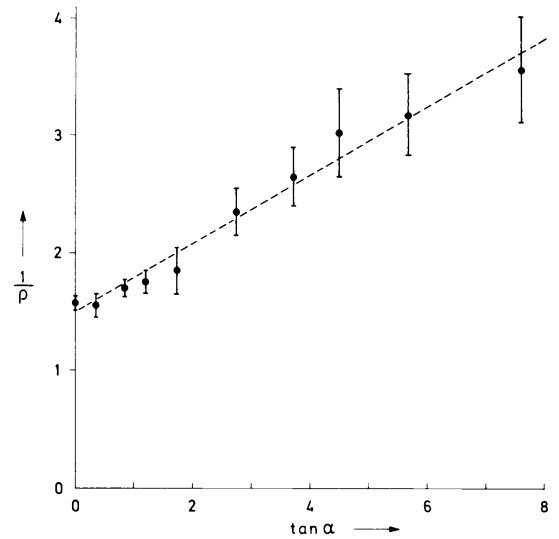

Fig. 4. Reciprocal density $1 / \rho$ versus tana. The dotted line represents the relation $\rho=1 /(p+q \tan \alpha)$ with $p=1.50$ and $q=0.291$.

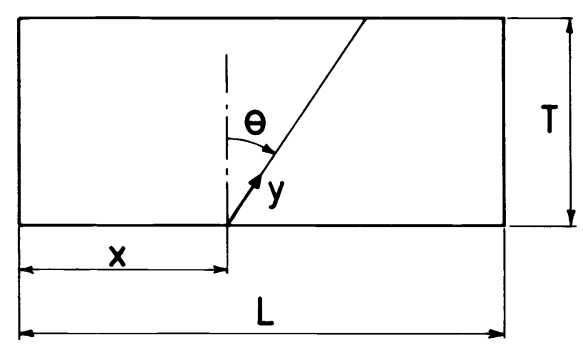

Fig. 5. Coordinates used for calculations.

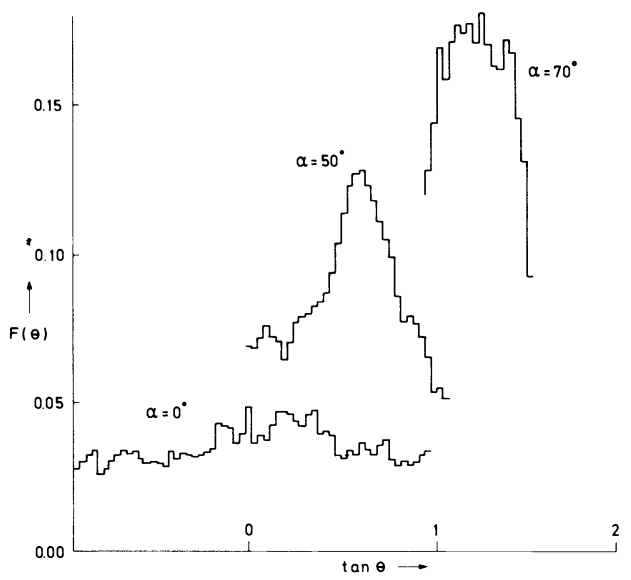

Fig. 6. Column orientation function $F(\theta)$ for three simulated films.

observed that $F(\theta)$ for the film deposited at normal incidence does not exhibit a clear maximum. This is apparently due to the large number of branches of the columns, as can be observed in Fig. 2. At off-normal angles that are not too small, $F(\theta)$ exhibits a more pronounced peak from which $\beta$ can be estimated. The values of $\tan \beta$ obtained are shown in Fig. 7 as a function of $\tan \alpha$.

At angles that are not too high, the dependence of $\beta$ on $\alpha$ can be described by

$\tan \beta=\frac{1}{2} \tan \alpha$. 


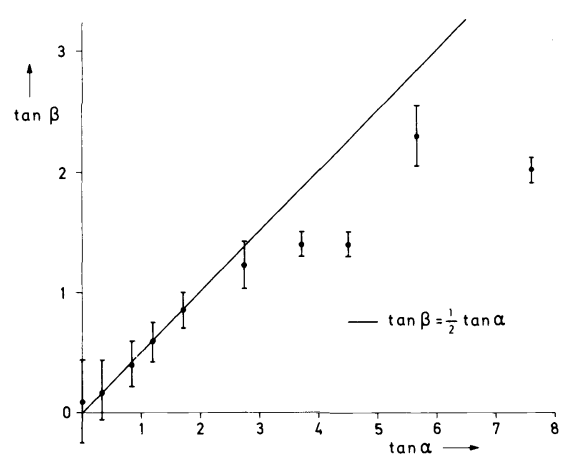

Fig. 7. Tangent of column angle $\beta$ versus $\tan \alpha$ for all simulated films.

This relation has been proposed before ${ }^{17,20}$ and is confirmed by experimental observations up to deposition angles of $80^{\circ} .14,17,18$ In our case, however, Eq. (3) apparently does not hold above $\tan \alpha=3\left(\alpha=70^{\circ}\right)$. The reason for this discrepancy may be the finiteness of the film segment in combination with the periodic boundary conditions imposed on the $\mathrm{x}$-coordinate.

This seems to be confirmed by the results mentioned in the next section, where we see that the "natural" period of the structure approaches the length $\mathrm{L}$ of the simulated film segment.

\subsection{Periodicity}

To analyze the periodicity of the microstructure, the autocorrelation function $\mathrm{G}(\mathrm{z})$ is used:

$\mathrm{G}(\mathrm{z})=\frac{1}{\mathrm{~L}} \int_{0}^{\mathrm{L}}\left[\rho_{\mathrm{c}}(\mathrm{x})-\rho\right]\left[\rho_{\mathrm{c}}(\mathrm{x}+\mathrm{z})-\rho\right] \mathrm{dx}$,

where $\rho_{\mathrm{c}}(\mathrm{x})$ is the average density along a line parallel to the columns (see Fig. 5):

$\rho_{\mathrm{c}}(\mathrm{x})=\frac{\cos \beta}{\mathrm{T}} \int_{0}^{\mathrm{T} / \cos \beta} \rho(\mathrm{x}, \mathrm{y}) \mathrm{dy} \quad(\theta=\beta)$.

Again, only rows 8 through 39 are used in the calculation.

Figure 8 shows $\mathrm{G}(\mathrm{z} / \mathrm{L})$ for a film deposited at $\alpha=70^{\circ}$. The periodicity of the structure is clearly reflected in the behavior of $G(z / L)$. The length of the period can be roughly estimated by taking the distance between two maxima or minima.

Figure 9 depicts the dependence of the period on the deposition angle. Due to the large uncertainties in the estimated period, it is difficult to draw conclusions from this behavior. However, using the density results, we can test the hypothesis that the column width is independent of the deposition angle. It easily can be shown that in this case, assuming the density of the voids to be zero, the period should exhibit exactly the same behavior as $1 / \rho$. In Fig. 9, a set of lines is plotted for which the hypothesis is satisfied. It appears that at angles that are not too high, the hypothesis of a constant column width could very well be true. At high angles, deviations can be expected because the "natural" period interferes with the imposed periodic boundary conditions. This is illustrated in the figure, where the allowed values of the period of strictly periodic functions are indicated.

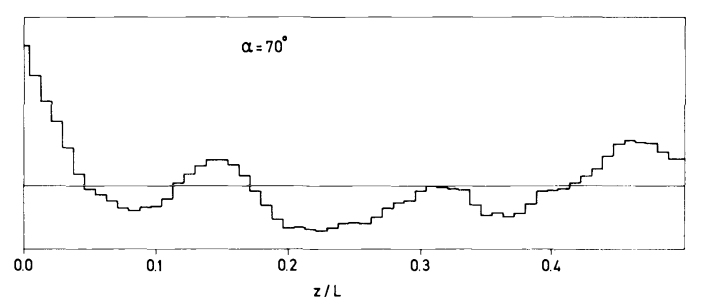

Fig. 8. Autocorrelation function $G(z / L)$ for a simulated film.

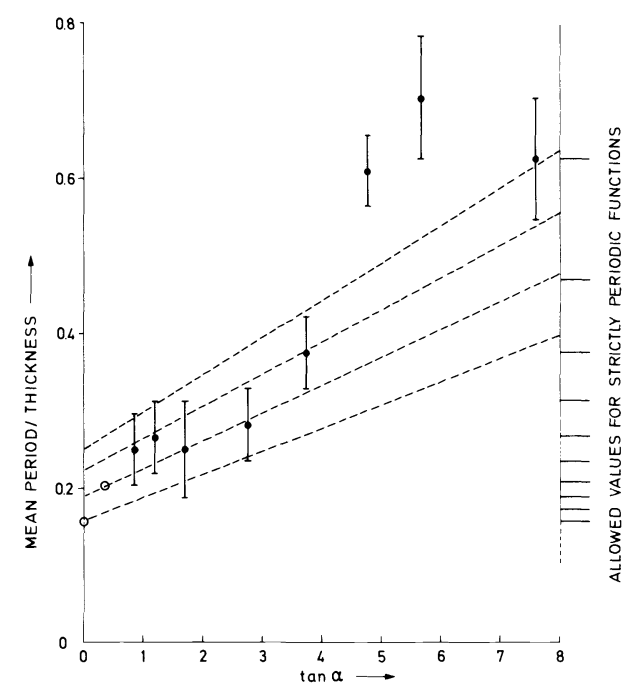

Fig. 9. Period of the structure versus tan $\alpha$ for all simulated films. The points indicated by the open circles are extremely uncertain.

\section{SIMPLIFIED MODEL FOR THE MICROSTRUCTURE OF A THREE-DIMENSIONAL FILM}

Based on the results mentioned in the previous paragraphs, we propose a simplified model in which the two-dimensional film is described in terms of a periodic array of dense columns $(\rho=1)$ with a constant width, separated by empty voids $(\rho=0)$ with a width $\mathrm{V}$ depending on the deposition angle:

$\mathrm{V}=\mathrm{V}_{\mathrm{o}}+\mathrm{V}^{\prime} \tan \boldsymbol{\alpha}$.

Realizing that the "shadow" of any formation of already condensed particles is simply proportional to $\tan \alpha$, this dependence strongly suggests that the void widening is a purely geometrical effect. The constant contribution can be interpreted as being the shadowing effect of a single particle due to its finite dimensions measured parallel to the substrate. As we already have observed, the void widening is consistent with the dependence of the density and the structure period on $\tan \alpha$ both in the simulated films and in real films. The column angle $\alpha$ still obeys Eq. (3): $\tan \beta=1 / 2 \tan \alpha$. This relation also can be explained on the basis of simple geometrical arguments, as has been pointed out before. ${ }^{17}$

The model can be extended to three dimensions if we assume that the two-dimensional model still holds for cross sections both parallel (effective angle of incidence $=\alpha$ ) and perpendicular (effective angle of incidence $=0$ ) to the plane through the incident vapor beam and substrate normal. In this case, it seems plausible to write, for the void width in any vertical cross section making an angle $\theta$ with the projected 


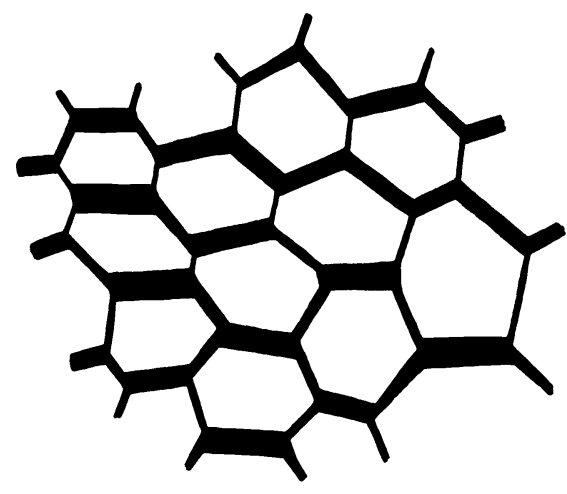

Fig. 10. Schematic representation of a horizontal cross section through a film, showing columns (white) and voids (black). The arrow indicates the vapor direction.

vapor beam,

$\mathrm{V}=\mathrm{V}_{\mathrm{o}}+\mathrm{V}^{\prime} \tan \alpha \cos \theta$

This dependence on $\theta$ was proposed earlier by Leamy, Gilmer, and Dirks. ${ }^{17}$

In a cross section parallel to the substrate, the columnar structure will look like Fig. 10, in which the widest voids run perpendicular to the projected vapor beam direction. ${ }^{17}$

\section{WATER PENETRATION IN OBLIQUELY DEPOSITED FILMS}

The columnar structure of thin films leads to considerable adsorption of water on the void surface and, at larger values of the humidity, to capillary condensation of water. ${ }^{21}$ It has been observed that the water penetrates preferentially through large defects into multilayer coatings and then spreads laterally. Figure 11 illustrates the water-penetrated areas in an $\mathrm{Ag} / \mathrm{SiO}_{\mathrm{x}} / \mathrm{Ag}$ filter; the water affects the effective refractive index and shifts the design wavelength of the filter, making it possible to observe the water-penetrated areas under monochromatic illumination. A number of $\mathrm{Ag} / \mathrm{SiO}_{\mathrm{x}} / \mathrm{Ag}$ filters have been prepared in which the $\mathrm{SiO}_{\mathrm{x}}$ layer is deposited at angles of $23^{\circ}, 30^{\circ}$, and $38^{\circ}$. In these filters the observed patches are elliptical, as is illustrated in Fig. 11(b). In all of these filters it is observed that the axial ratio of the patches is about 3:2 and that the long axis is oriented perpendicular to the vapor incidence plane.

A simple explanation of these results can be obtained if it assumed that the velocity of water transport $\mathrm{v}$ is proportional to the void width $\mathrm{V}$. In that case, the projected velocities in the $\mathrm{x}$-direction, normal to the vapor incidence plane, and the $\mathrm{y}$-direction, parallel to that plane, are proportional to $\mathrm{V} \cos \theta$ and $V \sin \theta$, respectively. Using Eq. (7) and averaging over randomly oriented voids yields for the average velocities $\bar{v}_{\mathbf{x}}$ and $\bar{v}_{\mathbf{y}}$

$\overline{\mathrm{v}}_{\mathrm{x}}=\mathrm{k}\left(2 \mathrm{v}_{\mathrm{o}}+\mathrm{V}^{\prime} \frac{\pi}{2} \tan \alpha\right)$,

$\overline{\mathrm{v}}_{\mathrm{y}}=\mathrm{k}\left(2 \mathrm{v}_{\mathrm{o}}+\mathrm{V}^{\prime} \tan \alpha\right)$,

where $\mathbf{k}$ is a proportionality constant

From these expressions it becomes clear that for $\tan \alpha \ll V_{o} / V^{\prime}$ we have $\bar{v}_{x}=\bar{v}_{y}$ and, therefore, the patches are circular. On the other hand, for $\tan \alpha \gg V_{0} / V^{\prime}$, we find (a)

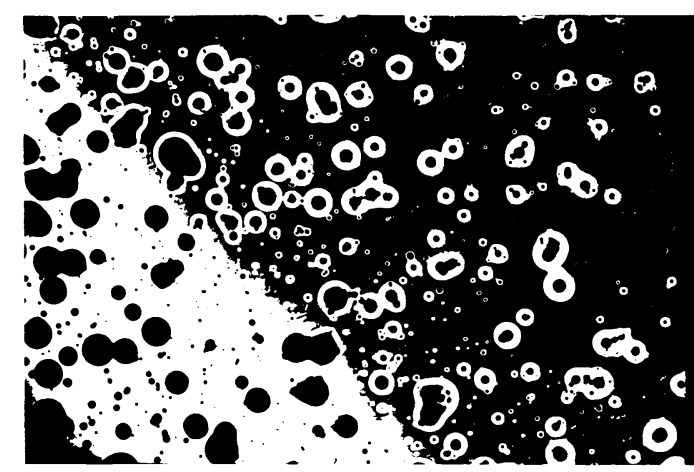

(b)

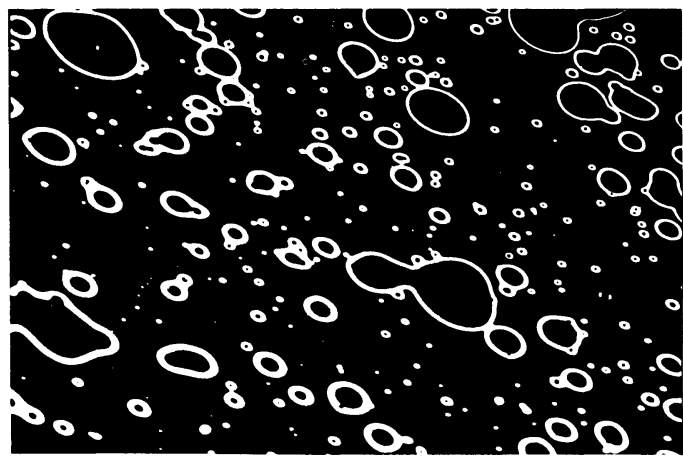

4

Fig. 11. Water penetration patches in an $\mathrm{Ag} / \mathrm{SiO}{ }_{x} / \mathrm{Ag}$ filter with $\mathrm{SiO}_{\mathrm{x}}$ incident (a) normally and (b) at $30^{\circ}$ in the arrow direction.

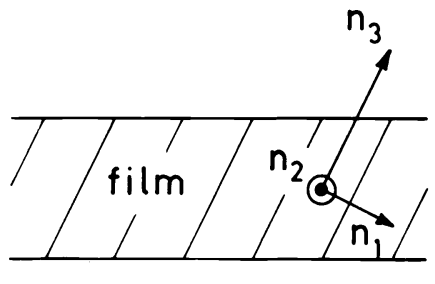

substrate

Fig. 12. Axes chosen for the refractive indices.

$\overline{\mathrm{v}}_{\mathrm{x}} / \overline{\mathrm{v}}_{\mathrm{y}}=\pi / 2$, and the patches become elliptical with axial ratio $\pi: 2$, oriented with their long axis perpendicular to the vapor incidence plane.

This model fully explains the experimental observations, indicating that our simplified microstructure model is valid for these films.

\section{FORM BIREFRINGENCE IN THIN FILMS}

As a result of the columnar structure, thin films will exhibit biaxial birefringence, and, consequently, the films are characterized by three indices of refraction. Figure 12 illustrates the choice of the axes for these indices.

The refractive indices for such a material are given by ${ }^{22}$

$\mathrm{n}_{\mathrm{j}}^{2}=\epsilon_{\mathrm{c}}-\frac{\mathrm{f}\left(\epsilon_{\mathrm{c}}-\epsilon_{\mathrm{v}}\right)}{1-\left[(1-\mathrm{f})\left(\epsilon_{\mathrm{c}}-\epsilon_{\mathrm{v}}\right) / \epsilon_{\mathrm{c}}\right] \mathrm{L}_{\mathrm{j}}} \quad(\mathrm{j}=1,2,3)$,

where $f$ is the void volume fraction $(f \ll 1), \epsilon_{\mathrm{c}}$ and $\epsilon_{\mathrm{v}}$ are the 
dielectric constants of the column and void materials, respectively, and $L_{j}$ is the depolarization factor of the voids in the corresponding direction $\left(\mathrm{L}_{1}+\mathrm{L}_{2}+\mathrm{L}_{3}=1\right)$.

If the void width is much smaller than the film thickness, which is a realistic assumption, it is clear that $\mathrm{L}_{3} \approx 0$. Further, if our microstructure model is valid, there is on the average a larger volume fraction of voids running perpendicular to the vapor incidence plane than parallel to that plane. Hence, we have $L_{2}<0.5$ and $L_{1}>0.5$. In that case, we can conclude from Eq. (9) that $n_{1}<n_{2}<n_{3}$.

To verify this result on experimental films, a number of different films have been investigated with an ellipsometric technique. This technique has been described before ${ }^{23}$ and involves four measurements to obtain the film thickness and the three indices of refraction. For example, for a $\mathrm{ZrO}_{2}$ film deposited at $30^{\circ}$, it is found that $n_{1}=1.948, n_{2}=1.966$, and $\mathrm{n}_{3}=2.033$. More detailed results were published elsewhere..$^{23}$ In all cases it is found that $n_{1}<n_{2}<n_{3}$, in agreement with the prediction.

Quantitative calculations of the indices have not been attempted so far. However, from the microstructure model and Eq. (9), the difference between the indices can be estimated and is found to be in the order of a few percent, which agrees with the experimental results.

\section{CONCLUSIONS}

In this paper, it has been demonstrated that computer simulations are useful to obtain insight into the origin and nature of the typical columnar structure of vapor-deposited thin films. Apparently, the major factors determining the columnar growth are the limited mobility of the condensing atoms or molecules and the geometrical "shadowing" effect. It has been found from a series of simulations that the structure can be described in terms of columns with a size independent of the vapor incidence angle, separated by voids that become wider with increasing angle of incidence. This simplified picture can be extended to three dimensions if it is assumed that the two-dimensional model is still valid for cross sections both perpendicular and parallel to the vapor incidence plane. Predictions of the anisotropy of water-penetration fronts and optical birefringence in thin films, based on this model, are found to agree with experimental results on obliquely deposited thin films. Further work will include the improvement of the statistics of the simulation by increasing the number of particles to about 25,000 . This already has been done for simulations at normal incidence but still needs to be done at oblique angles. Also, the effect of particle mobility on the results will be studied by using different relaxation schemes.

\section{ACKNOWLEDGMENTS}

We are grateful for the support of the Department of Defense under contract No. N60530-81-C-0158. We also acknowledge the assistance of C. C. Lee, R. Sprague, R. H. Potoff, and M. R. Jacobson. M. Sikkens gratefully acknowledges the support of the Netherlands Organization for the Advancement of Pure Research (ZWO).

\section{REFERENCES}

1. H. A. Macleod, in Optical Thin Films, R. I. Seddon, ed., Proc. SPIE Vol. 325, 21 (1982).

2. T. Hashimoto, K. Hara, K. Okamoto, and H. Fujiwara, J. Phys. Soc. Jpn. 41, 1433 (1976).

3. H. J. Leamy and A. G. Dirks, J. Appl. Phys. 50, 2871 (1979).

4. K. Ozawa, T. Yanada, H. Masuya, M. Sato, S. Ishio, and M. Takahashi, J. Magn. Mater. 35, 289 (1983).

5. F. L Galeener, Phys. Rev. Lett. 27, 1716 (1971)

6. T. M. Donovan, W. E. Spicer, J. M. Bennet, and E. J. Ashley, Phys. Rev. B2, 397 (1970).

7. M. Harris, H. A. Macleod, S. Ogura, E. Pelletier, and B. Vidal, Thin Solid Films 57, 173 (1979).

8. D. K. Pandya, S. K. Barthwal, and K. L. Chopra, Phys. Stat. Sol. (a) 32, 489 (1975).

9. B. A. Orlowski, W. E. Spicer, and A. D. Baer, Thin Solid Films 34, 31 (1976).

10. L. Kubler, G. Gewinner, J. J. Koulmann, and A. Jaéglé, Phys. Stat. Sol. (b) 78,149 (1976)

11. K. Okamoto, T. Hashimoto, K. Hara, and E. Tatsumoto, J. Phys. Soc. Jpn. 31, 1374 (1971).

12. J. D. Finegan and R. W. Hoffman, J. Appl. Phys. 30, 597 (1959).

13. H. K. Pulker and J. Maser, Thin Solid Films 59, 65 (1979)

14. A. G. Dirks and H. J. Leamy, Thin Solid Films 47, 219 (1977).

15. D. J. Henderson, M. H. Brodsky, and P. Chaudhari, Appl. Phys. Lett. 25, 641 (1974).

16. S. Kim, D. J. Henderson, and P. Chaudhari, Thin Solid Films 47, 155 (1977).

17. H. J. Leamy, G. H. Gilmer, and A. G. Dirks, in Current Topics in Materials Science, Vol 6, E. Kaldis, ed., North-Holland, Amsterdam, (1980).

18. N. G. Nakhodkin and A. I. Shaldervan, Thin Solid Films 10, 109 (1972).

19. D. K. Pandya, A. C. Rastogi, and K. L. Chopra, J. Appl. Phys. 46, 2966 (1975).

20. J. M. Nieuwenhuizen and H. B. Haanstra, Philips Tech. Rev. 27, 87 (1966).

21. H. A. Macleod and D. Richmond, Thin Solid Films 37, 163 (1976).

22. E. C. Stoner, Philos. Mag. 36, 803 (1945).

23. F. Horowitz, Ph.D. Dissertation, University of Arizona, Tucson (1983), 